\title{
Ecuaciones inversas de filtros de reconstrucción perfecta
}

\author{
Oscar Herrera-Alcántara ${ }^{1}$, María del Carmen E. Macías-Sánchez ${ }^{1}$, \\ Miguel González-Mendoza ${ }^{2}$ \\ 1 Universidad Autónoma Metropolitana, Azcapotzalco, México, \\ México \\ 2 Instituto Tecnológico y de Estudios Superiores de Monterrey, México, \\ México \\ oha@correo.azc.uam.mx, marycar81@hotmail.com, mgonza@itesm.mx
}

Resumen. En este trabajo presentamos las ecuaciones de filtros ortogonales de reconstrucción perfecta dependientes de un solo parámetro para el caso de longitud 4, así como sus ecuaciones inversas. Reportamos resultados experimentales que usan las ecuaciones inversas a fin de recuperar los valores de los parámetros. Los experimentos indican que sí es posible determinar los valores de los parámetros con alta precisión.

Palabras clave: wavelets, ecuaciones inversas de filtros paramétricos, respuesta al impulso, reconstrucción perfecta.

\section{Inverse Equations of Perfect Reconstruction Filters}

\begin{abstract}
We present the equations of four length perfect reconstruction orthogonal filters that depend of a single parameter, as well as their inverse equations. We report experimental results where the parameters are recovered with minimal error by applying the inverse equations.
\end{abstract}

Keywords: wavelets, inverse equations of parametric filters, response to the impulse, perfect reconstruction.

\section{Introducción}

Uno de los resultados más interesantes en el ámbito de la Transformada Wavelet Discreta (TWD) diádica es que se puede implementar con un banco de filtros que consta de un filtro pasabajas y un filtro pasaaltas. Estos filtros ortogonales de reconstrucción perfecta (FORP) suelen expresarse mediante un conjunto de coeficientes reales. Ejemplo de ello es el filtro Haar [12] de longitud dos, en donde el filtro pasabajas es $h=\left(h_{0}, h_{1}\right)=\left(\frac{1}{2}, \frac{1}{2}\right)$ y el filtro pasaaltas 
es $g=\left(g_{0}, g_{1}\right)=\left(\frac{1}{2},-\frac{1}{2}\right)$. El conjunto de coeficientes de $h$ y $g$ representan la respuesta al impulso de los filtros (en el dominio temporal), que corresponden respectivamente a un filtro pasabajas y a un filtro pasaaltas (en el dominio de la frecuencia).

Un FORP de mayor longitud involucra un número par de coeficientes, y existe un número infinito de filtros que cumplen la propiedad de que al aplicarlos apropiadamente a una señal permiten recuperarla fielmente. La etapa de análisis de la señal consiste en aplicar los filtros $h$ y $g$, en tanto que la fase de síntesis consiste en aplicar variantes de los filtros $h$ y $g$ al resultado de la fase de análisis, por lo que se puede decir que las fases de análisis y síntesis son procesos inversos.

En la práctica, la señal original y la reconstruida en la fase de síntesis, difieren en el error de precisión de la aritmética de punto flotante, pero en la literatura se les conoce como filtros de reconstrucción perfecta (FRP). Si además cumplen el criterio de ortogonalidad, se les llama filtros ortogonales de reconstrucción perfecta (FORP). Se han publicado varios FRP como los Daubechies, los Coiflets y los Symlets [1][2][14]. Y también se han publicado ecuaciones paramétricas de filtros que permiten generar FRP $[11,16,15,13,8,10]$.

Sin embargo, continúa siendo relevante la siguiente pregunta: Dados los coeficientes de un FRP ¿qué parámetros le corresponden?

De esta forma, en el presente trabajo presentamos las ecuaciones inversas de FORP que devuelven el valor de los parámetros a partir de los coeficientes de la respuesta al impulso, específicamente de filtros de longitud 4.

La parametrización de FORP relacionados con funciones wavelets es de interés porque permite reducir el espacio de búsqueda en conjuntos de coeficientes que cumplan con la condición de reconstrucción perfecta, y toda vez que la construcción de wavelets como bases de funciones es relevante, tanto como lo son las funciones seno y coseno para el análisis de Fourier, solo que en este último las bases son exclusivamente senos y cosenos, y su forma está claramente definida, en tanto que en el análisis wavelets se pueden tener infinitas funciones base, muchas de ellas ligadas a FRP.

Como ya se mencionó, se han propuesto varios métodos para calcular FRP, de los cuales uno se debe a I. Daubechies [1] quien presentó filtros que generan funciones wavelet con el máximo número de sus momentos de desvanecimiento, los cuales se reflejan en la maximización de la regularidad de las funciones wavelet asociadas, es decir, generan las wavelets más "suaves" posiblemente construibles para una longitud de filtro dada.

Otro método de diseño de FRP es el algebraico [7], en donde se propone resolver ecuaciones derivadas de las condiciones de reconstrucción perfecta. El método algebraico da lugar a ecuaciones paramétricas que, para ciertos valores de parámetros, dan cabida a los FORP presentados por I. Daubechies.

Cabe mencionar que las ecuaciones inversas de FRP paramétricos de [7] ya se han aplicado, por ejemplo, en compresión de imágenes $[3,4,5]$. Lo que en este trabajo presentamos es la metodología de cómo obtener las ecuaciones inversas, una técnica de reparación para que los parámetros se mantengan en el intervalo 
$[0,2 \pi)$, y estudios experimentales de algunas de sus propiedades, considerando los resultados obtenidos en [9].

El artículo está organizado de la siguiente manera, en la Sección 2 presentamos la metodología seguida en el presente trabajo de investigación, en la Sección 3 revisamos el método algebraico para obtener las ecuaciones paramétricas, en la Sección 4 calculamos las ecuaciones inversas de filtros de longitud 4, en la Sección 5 presentamos varios experimentos y sus resultados, y finalmente, en la Sección 6 presentamos conclusiones y trabajos futuros.

\section{Metodología}

En el estudio de las ecuaciones inversas de los FORP seguimos los siguientes pasos:

1. Revisamos las ecuaciones paramétricas que generan coeficientes de FORP,

2. Realizamos cálculos para despejar los parámetros, a partir de las ecuaciones del Paso 1, y les llamamos ecuaciones inversas,

3. Proponemos una técnica de reparación de los valores de los parámetros obtenidos de las ecuaciones inversas, de acuerdo a la periodicidad de las funciones seno y coseno, y a las propiedades de las funciones trigonométricas arco seno y arco coseno,

4. Realizamos experimentos con un programa de computadora para medir los errores de aproximación entre de los parámetros originales y los coeficientes recuperados con ecuaciones inversas.

\section{Parametrización de filtros de reconstrucción perfecta}

La parametrización de FORP ha sido abordada en trabajos previos. Además, se han propuesto varias parametrizaciones, así como métodos de parametrización. En [11] se discute la parametrización de coeficientes de funciones de escalamiento (ortogonales a las funciones wavelet) con varios momentos de desvanecimiento. En [16] se presentan parametrizaciones de wavelets ortonormales con soporte en el intervalo $[0,2 N-1)$ en donde $N$ es un entero par que involucra $N-1$ parámetros que varían en el intervalo $[0,2 \pi)$.

En cuanto a aplicaciones de las ecuaciones paramétricas, en [3] se utilizan parametrizaciones de filtros y se presenta una técnica para calcular la mejor wavelet para una imagen dada. La técnica usada, calcula la relación señal a ruido y ajusta los coeficientes de los filtros (y por ende las funciones wavelet) dinámicamente para mejorar el desempeño en una aplicación de codificación. En [7] se presenta un método algebraico para parametrizar filtros de longitud 4, 6, 8 y 10 , y se presentan valores específicos de parámetros que corresponden a los filtros publicados por I. Daubechies.

En [4] se aplica un algoritmo genético para determinar el conjunto de parámetros de filtros de longitud 4,6,8 y 10 en donde la función de aptitud maximiza 
la cantidad de coeficientes wavelet despreciables, que generen el menor error de reconstrucción en imágenes en escalas de grises.

Ahora, el problema que abordamos consiste en lo siguiente: Dado un conjunto de coeficientes de filtros de reconstrucción perfecta conocer qué parámetros le corresponden.

Al respecto podemos mencionar que en [6] se presentan algunas ecuaciones paramétricas inversas que procuran responder la pregunta anterior, sin embargo, no se contempla el caso en el que los parámetros puedan estar fuera del rango $[0,2 \pi)$, y que por tanto requieran un "mecanismo de reparación". Dado que este problema aún no ha sido ampliamente abordado, al menos tanto como el estudio de parametrizaciones, y tampoco se ha profundizado en la existencia de múltiples soluciones y sus propiedades, lo hemos tomado como motivación para esta investigación.

De esta forma, trabajamos con filtros de longitud 4, y estudiamos varias alternativas de solución para calcular los parámetros. También se propone un mecanismo de "reparación" para que los parámetros obtenidos con las ecuaciones inversas queden nuevamente en el rango $[0,2 \pi)$, tal como se asume en las ecuaciones paramétricas.

\section{Ecuaciones inversas de filtros paramétricos}

La parametrización para un cierto filtro no es única y, además, para una parametrización dada se pueden generar varias opciones de ecuaciones inversas, las cuales ponen el valor de los parámetros en función de los coeficientes de la respuesta al impulso. Por ejemplo, para el filtro de longitud 4 se tienen cuatro coeficientes $h_{0}, h_{1}, h_{2}$ y $h_{3}$ dados por:

$$
\begin{aligned}
& h_{0}=\frac{1}{4}+\frac{\cos \alpha}{2 \sqrt{2}}, \\
& h_{1}=\frac{1}{4}+\frac{\sin \alpha}{2 \sqrt{2}}, \\
& h_{2}=\frac{1}{4}-\frac{\cos \alpha}{2 \sqrt{2}}, \\
& h_{3}=\frac{1}{4}-\frac{\sin \alpha}{2 \sqrt{2}} .
\end{aligned}
$$

Que dependen del parámetro $\alpha \in[0,2 \pi)$. De (1) a (4) se puede despejar $\alpha$ en cada una de ellas y obtener respectivamente:

$$
\begin{aligned}
& \alpha=a \cos \left(2 \sqrt{2}\left(h_{0}-\frac{1}{4}\right),\right. \\
& \alpha=a \sin \left(2 \sqrt{2}\left(h_{1}-\frac{1}{4}\right),\right. \\
& \alpha=a \cos \left(2 \sqrt{2}\left(\frac{1}{4}-h_{2}\right),\right.
\end{aligned}
$$




$$
\alpha=\operatorname{asin}\left(2 \sqrt{2}\left(\frac{1}{4}-h_{3}\right) .\right.
$$

Cada una de las ecuaciones (5) a (8) puede dar un valor diferente para el parámetro $\alpha$, inclusive ese valor puede estar fuera de rango $[0,2 \pi)$ dadas las propiedades de las funciones trigonométricas inversas involucradas. A continuación, revisamos el método de parametrización algebraica (ver [7]) para obtener las ecuaciones de FORP de longitud 4, para luego calcular sus respectivas inversas.

\subsection{Parametrización de filtros de longitud 4}

Se parte de la ecuación de reconstrucción perfecta

$$
\left|H_{n}(z)\right|^{2}+\left|H_{n}(-z)\right|^{2}=1
$$

en donde $H_{n}(z)=h_{0}+h_{1} z+h_{2} z^{2}+h_{3} z^{3}+\ldots+h_{n-1} z^{n-1}$ es un polinomio trigonométrico con $n$ coeficientes $h_{i}$, con $i=0,1,2, \ldots, n-1$. Para cuatro coeficientes se tiene que:

$$
H_{4}(z)=h_{0}+h_{1} z+h_{2} z^{2}+h_{3} z^{3} .
$$

Entonces, al desarrollar la ecuación (9) se obtiene:

$$
2\left(h_{0}^{2}+h_{2}^{2}+h_{1}^{2}+h_{3}^{2}\right)+2\left(h_{0} h_{2}+h_{1} h_{3}\right) z+2\left(h_{0} h_{2}+h_{1} h_{3}\right) z^{2}=1 .
$$

Y para lograr la reconstrucción perfecta se hace:

$$
h_{0}^{2}+h_{2}^{2}+h_{1}^{2}+h_{3}^{2}=\frac{1}{2}
$$

además de:

$$
h_{0} h_{2}+h_{1} h_{3}=0 .
$$

Al sumar (12) con (13) multiplicado por (-2) se obtiene el cuadrado perfecto

$$
\left(h_{0}-h_{2}\right)^{2}+\left(h_{1}-h_{3}\right)^{2}=\frac{1}{2} .
$$

Entonces, por analogía con la identidad trigonométrica $\sin ^{2} x+\cos ^{2} x=1$ se introduce un parámetro $\alpha$, y se deduce que:

$$
h_{0}-h_{2}=\frac{1}{\sqrt{2}} \cos \alpha
$$

y

$$
h_{1}-h_{3}=\frac{1}{\sqrt{2}} \sin \alpha
$$

Puesto que $H_{4}(z=1)=1$ entonces

$$
h_{0}+h_{1}+h_{2}+h_{3}=1
$$


y considerando que $H_{4}(z=-1)=0$ se obtiene

$$
h_{0}-h_{1}+h_{2}-h_{3}=0,
$$

entonces, sumando (17) con (18) se tiene

$$
h_{0}+h_{2}=\frac{1}{2}
$$

y substrayendo (18) de (17) se tiene

$$
h_{1}+h_{3}=\frac{1}{2}
$$

que, al combinarlas con (15) y (16), nos llevan a las ecuaciones paramétricas (ver ecuaciones (1) a (4)) del FORP de longitud cuatro:

$$
\begin{aligned}
& h_{0}=\frac{1}{4}+\frac{1}{2 \sqrt{2}} \cos \alpha, \\
& h_{1}=\frac{1}{4}+\frac{1}{2 \sqrt{2}} \sin \alpha, \\
& h_{2}=\frac{1}{4}-\frac{1}{2 \sqrt{2}} \cos \alpha, \\
& h_{3}=\frac{1}{4}-\frac{1}{2 \sqrt{2}} \sin \alpha .
\end{aligned}
$$

\subsection{Ecuaciones inversas para parametrizaciones de filtros de longitud 4}

Ahora, partiendo de las ecuaciones (21) a (24), determinaremos expresiones para $\alpha$ en términos de los coeficientes $h_{0}, h_{1}, h_{2}$ y $h_{3}$, a las cuales nos referimos como ecuaciones inversas de los FORP.

Para ello, nótese que al restar $h_{0}$ y $h_{2}$ se tiene:

$$
h_{0}-h_{2}=\frac{1}{\sqrt{2}} \cos \alpha,
$$

de donde se puede despejar $\alpha$, así:

$$
\alpha=\operatorname{acos}\left(\sqrt{2}\left(h_{0}-h_{2}\right)\right) .
$$

Restando $h_{1}$ y $h_{3}$ se tiene

$$
h_{1}-h_{3}=\frac{1}{\sqrt{2}} \sin \alpha,
$$

de donde también se puede despejar $\alpha$, entonces:

$$
\alpha=\operatorname{asin}\left(\sqrt{2}\left(h_{1}-h_{3}\right)\right) .
$$


Como se puede apreciar, existen al menos dos alternativas para calcular $\alpha$. Otras alternativas para calcular $\alpha$ son mediante el despeje directo en (21) a (24) de donde se obtienen respectivamente:

$$
\begin{aligned}
& \alpha=\operatorname{acos}\left(2 \sqrt{2}\left(h_{0}-\frac{1}{4}\right)\right), \\
& \alpha=\operatorname{asin}\left(2 \sqrt{2}\left(h_{1}-\frac{1}{4}\right)\right), \\
& \alpha=\operatorname{acos}\left(2 \sqrt{2}\left(\frac{1}{4}-h_{2}\right)\right), \\
& \alpha=\operatorname{asin}\left(2 \sqrt{2}\left(\frac{1}{4}-h_{3}\right)\right) .
\end{aligned}
$$

Obsérvese que en (26) y en (28) se involucran dos coeficientes de los FORP en cada ecuación, en tanto que en (29), (30), (31) y (32) se involucra un único coeficiente en cada ecuación, por lo que no esperaríamos que puedan devolver en todos los casos exactamente los mismos valores, y para ello realizaremos resultados experimentales con cada una de ellas en la Sección 5.

\subsection{Reparación de los parámetros}

Debido a que los valores de los parámetros $\alpha$ obtenidos con las ecuaciones paramétricas inversas no son necesariamente positivos, se propone una técnica de reparación, que consiste en que para cada valor de $\alpha$, se generen las variantes $v$ siguientes:

$$
\begin{gathered}
v=|\alpha|, \\
v=(\pi+|\alpha|), \\
v=(\pi-|\alpha|), \\
v=(2 \pi-|\alpha|), \\
v=\left(\frac{3}{2} \pi-\left|\alpha-\frac{1}{2} \pi\right|\right) .
\end{gathered}
$$

$\mathrm{Y}$ en los casos donde $v$ es positivo, aplicamos la ecuación $v=v \bmod 2 \pi$, en donde mod $2 \pi$ actúa como una generalización del módulo o residuo de la división entera (que aplica solo a números enteros positivos) pero en este caso se usa en una división con el número real $2 \pi$. Esto está justificado por la periodicidad de las funciones trigonométricas seno y coseno, y dado que las funciones trigonométricas inversas arco coseno y arco seno devuelven valores negativos y positivos. Para validar esta técnica de reparación, realizamos los experimentos de la Sección 5. 


\section{Experimentos y resultados}

Acorde con la metodología de la Sección 2, realizamos varios experimentos por computadora con los siguientes pasos:

1. Generar un número aleatorio para $\alpha$ en el intervalo $[0,2 \pi)$ y con ello los coeficientes del FORP correspondiente,

2. Aplicar las ecuaciones paramétricas inversas para calcular los valores de $\alpha$ con varias alternativas dadas por las ecuaciones inversas,

3. Aplicar la reparación de los valores de $\alpha$,

4. Con los valores reparados de $\alpha$ calcular nuevamente las respuestas al impulso de los FORP,

5. Comparar los valores de parámetros del paso 1, con los del paso 4, y medir el error RMS,

6. Repetir $N$ veces los pasos 1 a 6 para obtener errores promedio.

En los experimentos tomamos $N=30^{3}=27000$, por lo que error RMS promedio indicará el error de aproximación entre el parámetro $\alpha$ de referencia y el calculado con las ecuaciones inversas, para las $N=27,000$ iteraciones. Los seis experimentos realizados tomaron en cuenta las seis ecuaciones (26), (28), (29), (30), (31) y (32), las cuales se identifican como Experimento 1, Experimento 2, Experimento 3, Experimento 4, Experimento 5 y Experimento 6, respectivamente. En la Tabla 1, se muestran los resultados de los seis experimentos.

Tabla 1. Resultados experimentales con las ecuaciones inversas de filtros de longitud cuatro.

\begin{tabular}{|c|c|c|}
\hline Experimento & Ecuación paramétrica inversa & $\begin{array}{c}\text { Error RMS promedio entre } \alpha \text { y } \\
\alpha \text { recuperada con ecuaciones } \\
\text { inversas }\end{array}$ \\
\hline 1 & $\alpha=\operatorname{acos}\left(2 \sqrt{2}\left(h_{0}-\frac{1}{4}\right)\right)$ & $2.76087359 \mathrm{E}-30$ \\
\hline 2 & $\alpha=\operatorname{asin}\left(2 \sqrt{2}\left(h_{1}-\frac{1}{4}\right)\right)$ & $2.08134790 \mathrm{E}-29$ \\
\hline 3 & $\alpha=\operatorname{acos}\left(2 \sqrt{2}\left(\frac{1}{4}-h_{2}\right)\right)$ & $2.76075449 \mathrm{E}-30$ \\
\hline 4 & $\alpha=\operatorname{asin}\left(2 \sqrt{2}\left(\frac{1}{4}-h_{3}\right)\right)$ & $2.08134870 \mathrm{E}-29$ \\
\hline 5 & $\alpha=\operatorname{acos}\left(2 \sqrt{2}\left(h_{0}-h_{2}\right)\right)$ & $2.76104429 \mathrm{E}-30$ \\
\hline 6 & $\alpha=\operatorname{asin}\left(2 \sqrt{2}\left(h_{1}-h_{3}\right)\right)$ & $2.08135582 \mathrm{E}-29$ \\
\hline
\end{tabular}

De la Tabla 1 se puede ver que:

- Los errores RMS para los parámetros $\alpha$ son muy pequeños,

- Los RMS más grandes se dan en los Experimentos 2, 4 y 6 que difieren en un orden de magnitud $(E-30$ vs. $E-29)$ con los Experimentos 1,3 y 5 . 


\section{Conclusiones y trabajo futuro}

Los filtros ortogonales de reconstrucción perfecta tienen utilidad en el análisis tiempo-frecuencia de señales porque con ellos se puede calcular la transformada wavelet discreta, en forma rápida y eficiente.

Un tema de investigación relacionado con el diseño de filtros digitales de reconstrucción perfecta es conocer ecuaciones paramétricas, a partir de las cuales se puedan obtener funciones wavelets con características específicas para el análisis de señales, y el método algebraico provee una solución a este problema.

Hemos aportado en la investigación de cómo calcular el parámetro que le corresponde a un filtro de reconstrucción perfecta, y presentamos como caso particular el análisis de un filtro de longitud 4 con un único parámetro, sin embargo, la metodología desarrollada puede aplicarse a filtros de mayor longitud.

Los resultados experimentales indican que sí es posible calcular los valores de los parámetros en el intervalo $[0,2 \pi)$ aplicando las ecuaciones inversas y una técnica de reparación validada experimentalmente.

Como trabajo futuro esperamos presentar más resultados con filtros de mayor longitud, y aplicaciones en el procesamiento de multimedios.

\section{Referencias}

1. Daubechies, I.: Ten Lectures on Wavelets. Society for Industrial and Applied Mathematics, Philadelphia, PA, USA (1992)

2. Daubechies, I.: Orthonormal bases of compactly supported wavelets ii. variations on a theme. SIAM Journal on Mathematical Analysis 24(2), 499-519 (1993)

3. Hereford, J., Roach, D., Pigford, R.: Image compression using parameterized wavelets with feedback (2003)

4. Herrera, O.: On the Best Evolutionary Wavelet Based Filter to Compress a Specific Signal. In: Advances in Soft Computing - 9th Mexican International Conference on Artificial Intelligence, MICAI 2010, Pachuca, Mexico, November 8-13, 2010, Proceedings, Part II. pp. 394-405 (2010)

5. Herrera, O., González, M.: Optimization of Parameterized Compactly Supported Orthogonal Wavelets for Data Compression. In: Advances in Soft Computing - 10th Mexican International Conference on Artificial Intelligence, MICAI 2011, Puebla, Mexico, November 26 - December 4, 2011, Proceedings, Part II. pp. 510-521 (2011)

6. Herrera, O., Mora, R.: Aplicación de Algoritmos Genéticos a la Compresión de Imágenes con Evolets. In: Avances Recientes en Sistemas Inteligentes. pp. 157-166. Sociedad Mexicana de Inteligencia Artificial (2011)

7. Lai, M.J., Roach, D.W.: Parameterizations of Univariate Orthogonal Wavelets With Short Support. Vanderbilt University Press (2002)

8. Lina, J.M., Mayrand, M.: Parametrizations for Daubechies wavelets. Phys. Rev. E 48, R4160-R4163 (Dec 1993), https://link.aps.org/doi/10.1103/PhysRevE.48.R4160

9. Macías, M.C., Herrera, O.: Cálculo de parámetros de filtros paramétricos de reconstrucción perfecta. Tech. rep., UAM (2016)

10. Pollen, D.: Parametrization of compactly supported wavelets. Aware, Inc. (AD890503.1.4) (1989) 
11. Regensburger, G.: Parametrizing compactly supported orthonormal wavelets by discrete moments. Applicable Algebra in Engineering, Communication and Computing 18(6), 583-601 (2007)

12. Resnikoff, H.L., Wells, R.O.: Wavelet Analysis: The Scalable Structure of Information. Springer-Verlag (1998)

13. Schneid, J., Pittner, S.: On the parametrization of the coefficients of dilation equations for compactly supported wavelets. Computing 51(2), 165-173 (1993)

14. Soman, K., Resmi, N., Ramachandran, K.: Insight Into Wavelets: from Theory to Practice. PHI Learning (2010)

15. Wells, R.: Parametrizing smooth compactly supported wavelets. Trans. Am. Math. Soc. 338(2), 919-931 (1993)

16. Zou, H., Tewfik, A.: Parametrization of compactly supported orthonormal wavelets. IEEE Trans. Signal Process. 41(3), 1428-1431 (1993) 